\title{
A closed-loop EKF and multi-failure diagnosis approach for cooperative GNSS positioning
}

\author{
Haiying Liu ${ }^{1,2}$, Xiaolin Meng ${ }^{2}$, Zhiming Chen ${ }^{1}$, Scott Stephenson ${ }^{2}$, Pekka Peltola ${ }^{2}$ \\ (1. College of Astronautics, Nanjing University of Aeronautics and Astronautics, Nanjing 210016, P. R. China \\ 2. Nottingham Geospatial Institute and Sino-UK Geospatial Engineering Centre, The University of \\ Nottingham, Nottingham, NG7 2TU, UK
}

\begin{abstract}
Current cooperative positioning with Global Navigation Satellite System (GNSS) for connected vehicle application mainly uses pseudorange measurements. However the positioning accuracy offered cannot meet the requirements for lane-level positioning, collision avoidance and future automatic driving, which needs real-time positioning accuracy of better than $0.5 \mathrm{~m}$. Furthermore, there is an apparent lack of research into the integrity issue for these new applications under emerging driverless vehicle applications. In order to overcome those problems, a new Extended Kalman Filter (EKF) and a multi-failure diagnosis algorithm are developed to process both GNSS pseudorange and carrier phase measurements. We first introduce a new closed-loop EKF with partial ambiguity resolution (PAR) as feedback to address the low accuracy issue. Then a multi-failure diagnosis algorithm is proposed to improve integrity and reliability. The core of this new algorithm includes using Carrier phase based Receiver Autonomous Integrity Monitoring (CRAIM) method for failure detection, and the double extended w-test detectors to identify failure. A cooperative positioning experiment was carried out to validate the proposed method. The results show that the proposed closed-loop EKF can provide highly accurate positioning, and the multi-failure diagnosis method is effective in detecting and identifying failures for both code and carrier phase measurements.
\end{abstract}

Keywords GNSS.cooperative positioning.

EKF.CRAIM-extended w-test

\section{Introduction}

To improve road transportation safety and efficiency, cooperative positioning by sharing *Corresponding author: haiying liu, email: liuhaiying@nuaa.edu.cn
Global Navigation Satellite System (GNSS) information among multi-vehicles has started to attract more attention in recent years (Alam and Dempster 2013; Bauernfeind et al. 2013; Stephenson et al. 2014). While the conventional GNSS positioning such as the differential GPS (DGPS) has been studied widely, the distinguished characteristics of cooperative positioning approach is the use of vehicle to vehicle (V2V) and vehicle to infrastructure (V2I) communications, for example the vehicle ad hoc networks (VANETs) (Alam and Dempster 2013; Stephenson et al. 2012, 2013). Liu et al. (2014) and Muller et al. (2014) addressed the issues in cooperative positioning with DGPS. Basnayake et al. (2011) used code and Doppler measurements to implement cooperative positioning. Those are rather simple cooperative positioning approach without the requirement of ambiguity resolution, but they sacrifice the high accuracy achievable from carrier phase measurements, so that the positioning accuracy and integrity cannot meet the requirements for lane-level positioning that requires positioning accuracy of better than $0.5 \mathrm{~m}$, lane departure warning, collision avoidance and future automatic driving for intelligent transportation applications (Stephenson et al. 2014).

In order to improve positioning accuracy in transport related applications several advanced approaches based on carrier phase measurements have been proposed (Liu et al. 2013; Schuster et al. 2012). Although GPS has been used for different civil positioning and navigation applications for more than three 
decades, the integer ambiguity resolution still remains a major challenge for carrier phased based solutions (Hofmann et al. 2008; Wang 2012). There are no known approaches that can estimate both real-valued navigation parameters and integer ambiguities at the same time. The prominent approaches perform a float estimate in the first step of least squares (LS) estimation, then use an integer fixing algorithm, such as the Least squares AMBiguity Decorrelation Adjustment (LAMBDA) to fix ambiguities (Teunissen and Verhagen 2009; Verhagen and Li 2012). However, those methods may degrade the accuracy because they failed to consider the dynamics and stochastic models of moving objects such as vehicles, thus complicating the integration with Extended Kalman Filtering (EKF) (Eugenio and Mirko 2013). An open-loop approach was proposed in several relative kinematic positioning applications (Wolfe et al. 2007) in which the EKF provides float ambiguity solutions, and then the LAMBDA method is used to fix them to integer values. However, this approach requires that the float ambiguities are sufficiently close to the exact integers, otherwise, the LAMBDA approach will not be able to fix ambiguities and the overall navigation accuracy will be degraded.

In addition to the accuracy, the positioning integrity and reliability are other important parameters. In aviation applications the integrity theory is derived, while in geodesy the reliability theory is employed (Knight et al. 2010b). Currently, most of the integrity monitoring methods for GNSS are based on code measurements, for example, the well known Receiver Autonomous Integrity Monitoring (RAIM) algorithms (Lin et al. 2014; Sabatini et al. 2013). The Advanced RAIM (ARAIM) (Walter et al. 2014) and Relative RAIM (PRAIM) (Yun and Kee 2014) were also proposed recently with the development of the new generation GNSS. However, until today they are still mainly based on code measurements without taking the advantages of using carrier phase measurements. A carrier phase based RAIM method (CRAIM) has been proposed recently (Feng et al. 2009) which can also be applied in Precise Point Positioning (PPP) (Jokinen et al. 2012). However, the proposed CRAIM method is only used to detect a failure rather than identify it. Hewitson and Wang (2007, 2010) present the detection, identification, and adaptation (DIA) procedure using w-test. However, the DIA procedure is aimed for code measurements using least-squares method rather than carrier phase based EKF filter. Meanwhile, current research on integrity and reliability is mainly based on the single failure assumption. However, a key problem to solve in cooperative systems is that there are many nodes, thus increasing the chances of multiple failures.

In order to improve the performance of cooperative positioning, two innovative approaches are proposed. First, a new closed-loop EKF is designed, which differs from traditional ones because the Partial Ambiguity Resolution (PAR) is performed separately from dynamic EKF filter. This allows the improvement in positioning accuracy while avoiding the necessity to fix the whole ambiguity vector in the closed-loop. Second, a new multi-failure diagnosis algorithm based on modified CRAIM and double extended w-test is further proposed to improve integrity and reliability, which is efficient for multi-failure detection and identification for carrier phase measurements. The authors intend to address the above issues when carrier phase measurements are used to deliver cooperative positioning in connected vehicle applications. The effectiveness of the 
algorithm is demonstrated by designing a V2V cooperative positioning experiment using a GNSS reference station, an electric train and a road vehicle.

\section{New closed-loop EKF approach for cooperative GNSS positioning}

The cooperative positioning of vehicles has been addressed by using differenced code techniques (Liu et al. 2014; Muller et al. 2014), which offer a simpler implementation. However, this means sacrificing the higher accuracy available from carrier phase measurements. In the following, we will present a new closed EKF for cooperative positioning based on GNSS raw measurements containing both code and carrier phase.

\section{EKF design for cooperative GNSS positioning}

The double difference (DD) technique among receivers and satellites is chosen since it can balance processing complexity, practicality and performance for real time implementation. By taking differences between two GNSS satellites $(p, s)$ and two receivers $(r, m)$ on frequency $j$, the DD code and carrier phase equations can be written as (Hofmann et al. 2008; Wang 2012)

$$
\begin{aligned}
& P_{r m, j}^{p s}=\rho_{r m}^{p s}+\mu_{j} I_{r m}^{p s}+T_{r m}^{p s}+e_{r m}^{p s} \\
& \lambda_{j} \phi_{r m, j}^{p s}=\rho_{r m}^{p s}-\mu_{j} I_{r m}^{p s}+T_{r m}^{p s}-\lambda_{j} N_{r m, j}^{p s}+\varepsilon_{r m}^{p s}
\end{aligned}
$$

where $P_{r m, j}^{p s}=\left(P_{r, j}^{p}-P_{r, j}^{s}\right)-\left(P_{m, j}^{p}-P_{m, j}^{s}\right)$ is the code DD on frequency $j$, and $\phi_{r m, j}^{p s}=\left(\phi_{r, j}^{p}-\phi_{r, j}^{s}\right)-\left(\phi_{m, j}^{p}-\phi_{m, j}^{s}\right) \quad$ represents the carrier phase DD. The symbol $\rho_{r m}^{p s}$ represents the combination of geometric distances between receivers and satellites, $I_{r m}^{p s}$ represents the ionosphere delay with coefficient $\mu_{j}=\left(\lambda_{j} / \lambda_{1}\right)^{2}, \quad \lambda_{j}$ is the wavelength for frequency $j, T_{r m}^{p s}$ represents the troposphere delay, $N_{r m, j}^{p s}$ is the DD ambiguity, and $e_{r m}^{p s}$ and $\varepsilon_{r m}^{p s}$ are error terms. In order to processing DD observations, the extended Kalman filtering (EKF) algorithm is adopted because it is efficient and suitable for real-time positioning with a large number of states and observations.

In order to address the issues as discussed in the introduction a new closed-loop EKF filter is designed as shown in Figure 1. Due to the high dynamic feature, a high order dynamics model is used in the EKF filter. The EKF is in charge of generating a float estimate; the state vector includes position $\widehat{\boldsymbol{b}}$, velocity $\dot{\overrightarrow{\boldsymbol{b}}}$, acceleration $\ddot{\vec{b}}$ and float ambiguities $\widehat{N}_{r m, j}^{p s}$. Starting from the float estimate, the partial ambiguity resolution (PAR) based on LAMBDA (Liu et al. 2013) is used to produce the fixed subset $\widehat{N}_{1}$ and the unfixed subset $\breve{N}_{2 \mid 1}$, which is used to fix the relative baseline vector $\breve{b}$. The partially fixed ambiguity $\breve{N}_{\text {PAR }}$ and the fixed baseline $\breve{b}$ are then fed back to the EKF filter to narrow down the solution in the following steps. The relative velocity $\dot{\vec{b}}$ and acceleration $\ddot{\vec{b}}$ can also be improved by the ambiguity resolution.

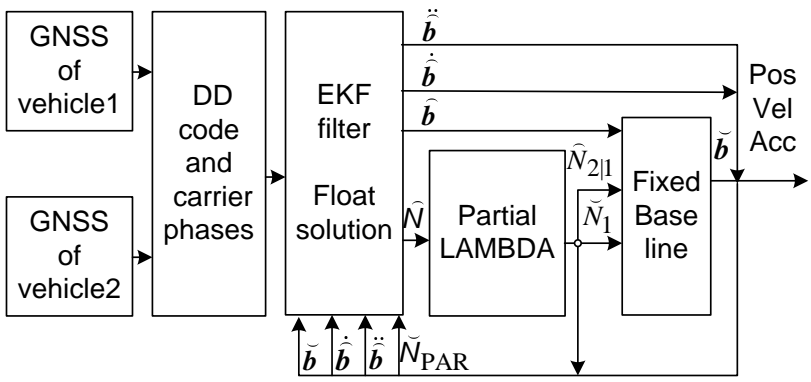

Fig. 1 New closed-loop architecture approach

According to the above discussion, the state vector $X$ in a closed-loop EKF filter is expressed as

$$
X=\left[\begin{array}{llll}
\boldsymbol{b} & \dot{\boldsymbol{b}} & \ddot{\boldsymbol{b}} & N_{r m, j}^{p s}
\end{array}\right]^{T}
$$

where $\boldsymbol{b}, \dot{\boldsymbol{b}}$ and $\ddot{\boldsymbol{b}}$ represent the position, velocity and acceleration vector respectively. $N_{r m, j}^{p s}$ represents the float ambiguities.

Based on the cooperative dynamic model, the 
matrix form of the system state equation is given by

$$
X_{k+1}=F X_{k}+G w_{k}
$$

where $X_{k+1}$ represents the state vector at epoch $k+1, \quad F$ represents the system dynamic matrix, $G$ is the system noise matrix, and $\boldsymbol{w}_{k}$ is the system noise vector with the covariance matrix $Q$. The state in the dynamic model can be adjusted by the matrix $F$ and $G \boldsymbol{w}_{k}$. In a constant acceleration motion model, $F$ is written as

$$
F=\left[\begin{array}{cccc}
I_{3 \times 3} & I_{3 \times 3} & 0 & 0 \\
0 & I_{3 \times 3} & I_{3 \times 3} & 0 \\
0 & 0 & I_{3 \times 3} & 0 \\
0 & 0 & 0 & I_{n \times n}
\end{array}\right]
$$

where $I_{n \times n}$ represents a $n \times n$ identity matrix, the zero matrices are of appropriate size, and $n$ is the number of the DD ambiguities.

The DD code and carrier phases given in (1) and (2) can be linearized from the EKF of cooperative positioning as

$Z_{k}=H_{k} X_{k}+v_{k}$

where $Z_{k}$ represents the measurement vector and $X_{k}$ is the state vector. The $H_{k}$ is the measurement matrix and $\boldsymbol{v}_{k}$ represents the measurement noise with covariance matrix $R$.

According to the state equation (4) and the observation equation (6), cooperative positioning can be calculated by the recursive EKF equations (Feng et al. 2009). For the initial estimation of $\hat{X}_{0}$, The Bancroft algorithm is used to initialize the relative position $\widehat{\boldsymbol{b}}_{0}$ with a meter level error. A least-squares adjustment is further used to reduce the initial positioning error. The initial ambiguities $\widehat{N}_{0}$ can be roughly obtained as

$\widehat{N}_{r m, j}^{p s}=\frac{\rho_{r m}^{p s}-\lambda_{j} \phi_{r m, j}^{p s}}{\lambda_{j}}+\frac{\varepsilon_{r m}^{p s}}{\lambda_{j}}$

where all of the symbols are defined in (2) and the residual atmospheric delays are disregarded.
The initial state covariance $Q$ is defined as

$$
Q=\left[\begin{array}{cccc}
\sigma_{\widehat{b}}^{2} & 0 & 0 & 0 \\
0 & \sigma_{\tilde{b}}^{2} & 0 & 0 \\
0 & 0 & \sigma_{\tilde{b}}^{2} & 0 \\
0 & 0 & 0 & \sigma_{\tilde{N}}^{2}
\end{array}\right]
$$

where $\sigma_{\vec{b}}^{2}, \sigma_{\hat{b}}^{2}, \sigma_{\overparen{b}}^{2}$ and $\sigma_{\widehat{N}}^{2}$ are the position, velocity, acceleration and ambiguities error variance respectively. The measurement noise covariance $R$ of the DD is given as

$$
R=\left[\begin{array}{cc}
R_{c} & 0 \\
0 & R_{p}
\end{array}\right]
$$

where $R_{c}$ and $R_{p}$ are noise covariance matrix of code DD and carrier phase DD respectively, which is given as

$$
R_{l}=\sigma_{l}^{2}\left[\begin{array}{cccc}
1 & 0.5 & \cdots & 0.5 \\
0.5 & 1 & \ddots & \vdots \\
\vdots & \ddots & \ddots & 0.5 \\
0.5 & \cdots & 0.5 & 1
\end{array}\right], \quad l=c, p
$$

where $\sigma_{c}^{2}$ and $\sigma_{p}^{2}$ are defined as the variance of the DD code and carrier phase respectively.

\section{Partial ambiguity resolution}

According to the practical applications of LAMBDA method, the probability of successfully fixing all the ambiguities is often low, especially in harsh environments. Furthermore, fixing all the ambiguities is unnecessary when a large number of satellites are visible. Thus the partial ambiguity resolution is used in the closed-loop EKF.

Once the PAR has been achieved, the resolution result $\breve{N}_{P A R}=\left[\begin{array}{ll}\breve{N}_{1} & \breve{N}_{2 \mid 1}\end{array}\right]$ is used to remove the ambiguous states from the state vector of EKF. It is also used to fix the relative baseline. The fixed baseline and its covariance are

$$
\breve{\boldsymbol{b}}=\widehat{\boldsymbol{b}}+\boldsymbol{P}_{\widehat{\boldsymbol{b}} \hat{N}} \boldsymbol{P}_{\widehat{N} \widehat{N}}^{-1}\left(\breve{N}_{\mathrm{PAR}}-\widehat{N}\right)
$$


$P_{b / \widetilde{N}_{\mathrm{PAR}}}=P_{\widehat{b} \bar{b}}-P_{\widehat{b} \hat{N}} P_{\bar{N} \hat{N}}^{-1} P_{\widehat{N} \bar{b}}$

where $P_{\widehat{N} \hat{N}}, P_{\widehat{b} \hat{N}}$ and $P_{\widehat{b} \hat{b}}$ are the corresponding sub-block of estimated state variance matrix $P$. Then $\breve{b}$ and $P_{b / \breve{N}_{\mathrm{PAR}}}$ are used recursively to update the state vector and covariance of EKF filter for the next epoch.

The new closed-loop approach differs from traditional LS method and the open-loop EKF method because a PAR algorithm is performed separately from the EKF filter. This avoids the necessity of fixing the whole ambiguity vector in the closed-loop. The PAR used in the feedback can improve the navigation accuracy and also ensure the smooth operation of the EKF in case of no fixed ambiguities.

\section{New multi-failure diagnosis algorithm for cooperative positioning}

Integrity reflects the ability to detect, identify and eliminate failures for continuous operation, which has become increasingly important in positioning systems especially in critical applications such as connected vehicles (Knight et al. 2010b). In aviation, the integrity theory is derived where the alarm time, continuity, integrity risk, etc. are emphasized. In geodesy, the reliability is employed where the internal and external reliability are more of concern. Although cooperative positioning for multi-vehicles is not identical to aviation or geodesy applications, essentially, to implement positioning integrity and reliability, the failure diagnosis including failure detection and identification should be resolved.

\section{New multi-failure diagnosis approach}

The research of failure diagnosis for carrier phase based positioning is still rare. Recently a CRAIM method is proposed to detect failure of carrier phase (Feng et al. 2009; Jokinen et al.
2012), but it cannot yet identify the failure. For failure identification a DIA procedure using w-test has been presented (Hewitson and Wang 2007, 2010). However, this method is aimed for code based positioning using least-squares model, rather than carrier phase based EKF model. Another problem is that the w-test was originally derived for single failure identification rather than multi-failure. However, in practical applications, the multi-failure diagnosis is required.

We propose a new multi-failure diagnosis algorithm based on the closed-loop EKF to detect and identify multiple failures of carrier phase based positioning. The new algorithm integrates a modified CRAIM and a newly extended w-test method, which is shown in Figure 2. First, the CRAIM method is modified for failure detection, in which a different test statistic is used to avoid confusion for the final judgment. Then two detectors using extended w-test are designed to identify failure of both code and carrier phase observations. Since the w-test was originally derived for single failure identification, a new approach is presented to extend the w-test for multi-failure.

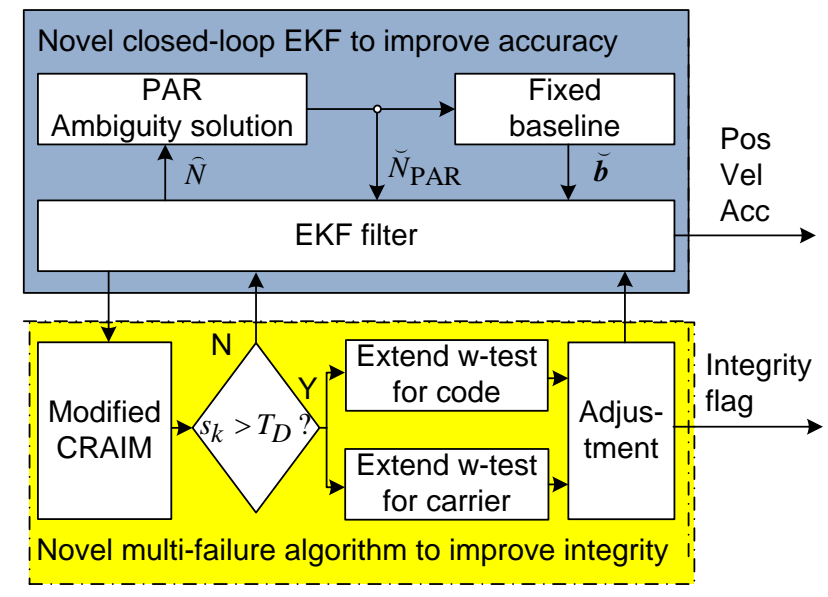

Fig. 2 New multi-failure diagnosis approach based on closed-loop EKF

In this new approach, the PAR algorithm, the modified CRAIM, and the double extended w-test detectors are integrated to the closed-loop EKF, as shown in the Figure 2. The 
role of each part is necessary and complementary to improve the accuracy and integrity for cooperative positioning. The PAR module increases the ambiguity resolution success rate, thus improving the accuracy and reliability for closed-loop processing. The modified CRAIM and the extended w-test are designed to detect and identify multiple failures. This approach not only improves the positioning integrity and reliability, but also has the advantages of computational efficiency and preserving the redundancy of the adjustment. The new algorithm is elaborated as following.

\section{The modified CRAIM for failure detection}

A vital factor for integrity monitoring is the innovation sequence of the EKF $\boldsymbol{r}_{k}$, which can be used to calculate test statistics values. At epoch $k$, it is expressed as

$\boldsymbol{r}_{k}=Z_{k}-H_{k} \hat{X}_{k \mid k-1}$

where $\hat{X}_{k \mid k-1}$ represents the predicted EKF state vector. The $\boldsymbol{r}_{k}$ provides the most relevant information for integrity monitoring, which is similar to the residual in the RAIM method. However, the measurement noise of DD observation is no longer independent as is shown in (10).

In the CRAIM method, the weighting matrix $W_{k}$ is used to de-correlate the dependence. Three different test statistics are carried out: the total test statistics $T_{a}$, code only $T_{c}$ and carrier phase only $T_{p}$, which are given as (Feng et al. 2009; Jokinen et al. 2012)

$$
\begin{aligned}
& T_{a}=\sqrt{\boldsymbol{r}_{k}^{T} W_{k}^{-1} \boldsymbol{r}_{k}} \\
& T_{c}=\sqrt{\boldsymbol{r}_{c k}^{T} R_{c}^{-1} \boldsymbol{r}_{c k}} \\
& T_{p}=\sqrt{\boldsymbol{r}_{p k}^{T} R_{p}^{-1} \boldsymbol{r}_{p k}}
\end{aligned}
$$

where $\boldsymbol{r}_{c k}$ and $\boldsymbol{r}_{p k}$ are the subsets of $\boldsymbol{r}_{k}$, corresponding to code and carrier phase DD.
The $R_{c}$ and $R_{p}$ are defined in (9). The weighting matrix $W_{k}$ takes into account the covariance, the measurement noise and their correlations of measurement residuals, which is given as

$W_{k}=H_{k} P_{k \mid k-1} H_{k}^{T}+R_{k}$

where $P_{k / k-1}$ represents the variance matrix of the predicted EKF state vector $\widehat{X}_{k \mid k-1}$.

However, in practical applications $T_{c}$ and $T_{p}$ only reflect the change of $\boldsymbol{r}_{k}$ as $R_{c}$ and $R_{p}$ are set in advance. Yet they have a great impact on $T_{c}$ and $T_{p}$; thus those different detection results may cause confusion for the final judgment.

Thus a modified CRAIM method is proposed, where only the total test statistics similar to (14a) is calculated, which is defined as

$s_{k}=\boldsymbol{r}_{k}^{T} W_{k}^{-1} \boldsymbol{r}_{k}$

where $s_{k}$ follows a $\chi^{2}$ distribution with a degree of freedom $n$ that equals to the number of measurements. It can be either a central or non-central $\chi^{2}$ distribution depending on the absence or presence of failure. Therefore, the corresponding threshold $T_{D}$ can be determined for a given probability of false alarm $P_{F A}$, that is $T_{D}=\chi_{P_{F A}}^{2}(n, 0)$. If $s_{k}$ is greater than $T_{D}$, it is assumed that failures exist, and vice versa.

\section{The extended w-test for multi-failure identification}

Similar to the definition of the w-test statistics for least squares model (Hewitson and Wang 2007, 2010), two w-test statistics for the EKF, i.e. $w_{c i}$ and $w_{p i}$, are presented for code DD and carrier phase DD respectively, which can be deduced as

$$
w_{l i}=\left|\frac{e_{i}^{T} R_{l}^{-1} \boldsymbol{r}_{l k}}{e_{i}^{T} R_{l}^{-1} W_{l k} R_{l}^{-1} e_{i}}\right|, \quad l=c, p
$$

where subscript $l=c$ represents the code DDs and $l=p$ represents the carrier phase DDs. The 
$e_{i}$ represents a unit vector, in which if the $i$ th component has a value of ' 1 ' means that the $i$ th measurement is being tested. The $R_{l}$ $(l=c, p)$ is the measurement noise covariance matrix defined in (9), and $R_{l}^{-1} \quad(l=c, p)$ represents the weight matrix of the measurements. The $W_{l k}(l=c, p)$ is the subset of $W_{k}$ that is defined in (15), which represents the variance covariance of the innovation sequence $\boldsymbol{r}_{l k}(l=c, p)$.

When no failures exist, $w_{l i} \quad(l=c, p)$ should be a standard normal distribution, whereas a non-central normal distribution shows the presence of a failure. When giving the significance level $\alpha$, then

$w_{l i} \leq N_{\alpha / 2}(0,1), \quad l=c \quad l$

Therefore, the test threshold can be calculated by $T_{l w}=N_{P_{F A} / 2}(0,1)$ using false alarm $P_{F A}$. If $w_{l i} \geq T_{l w}$, then a failure is detected in either the $i$ th code DD $(l=c)$ or the $i$ th carrier phase $\operatorname{DD}(l=p)$.

In the practical applications, correlations are often found in the test statistics, especially in carrier phase DD where there is a strong correlation. Due to the correlation, a failure in the DD measurement is likely to cause too many $w_{i}$ exceeding the test threshold, thus creating difficulty in distinguishing the real failure. The degree of correlation in test statistics $w_{l i}$ and $w_{l j}$ is determined through the correlation coefficient

$$
\begin{aligned}
& \rho_{l, i j}=\frac{e_{i}^{T} R_{l}^{-1} W_{l k} R_{l}^{-1} e_{j}}{\sqrt{e_{i}^{T} R_{l}^{-1} W_{l k} R_{l}^{-1} e_{i}} \cdot \sqrt{e_{j}^{T} R_{l}^{-1} W_{l k} R_{l}^{-1} e_{j}}}, \\
& l=c, p
\end{aligned}
$$

The two test statistics are fully correlated when $\rho_{l, i j}$ is equal to one, and they are completely uncorrelated when $\rho_{l, i j}$ is equal to zero. The greater the correlation between two test statistics, the more difficult it is to separate the corresponding measurements.

The w-test is originally derived for single failure identification. Knight et al. (2010a, 2010b) extended the w-test to the case of two failures, but the amount of failures needs to be known in advance, which is not realistic. An extended w-test method is proposed for multi-failure in Hewitson and Wang (2006). However, it is based on a least squares model for code measurements only rather than using EKF model for carrier phase measurements.

A new extended w-test is improved to identify multiple failures for both code and carrier phase DD based on the closed-loop EKF. In the extended w-test approach, the influence due to the correlation of the test statistic of identified failure on the remaining statistics is removed. A reduced subset of test statistics is obtained. The algorithm to reduce the w-test statistics is given as

$w_{t, l i}=w_{t-1, l i}-w_{t-1, \max } \cdot \rho_{l \max , l i}, \quad l=c, p$

where $w_{t, l i}$ is the reduced $\mathrm{w}$-test statistics of the $i$ th DD observation at the current iteration $t$. The $w_{t-1, i}$ is the test statistics of the $i$ th DD observation at the previous iteration $t-1$. The $w_{t-1, \max }$ is the maximum value of the test statistics at the previous iteration $t-1$. The $\rho_{l \text { max }, l i}$ is the correlation coefficient between $w_{t-1, l i}$ and $w_{t-1, \max }$. Using this algorithm, the influence of the largest failure on the remaining statistics can be removed iteratively.

The extended w-test procedure proposed is shown in Figure 3. The first failure is identified using (17), where the largest test statistic exceeding the test threshold $T_{l w}$ is identified as the failure. Then for $\mathrm{i}=1: l \mathrm{n}$, where $l \mathrm{n}$ is the current number of code DD statistics or carrier phase DD statistics, the influence of the identified failure on the other statistics is estimated and then subtracted using (20). The process is iterated until no more failures are identified. For multi-failure cases, the extended w-test is computationally more efficient. Rather than simply removing highly correlated 
measurements, the redundancy and geometry of the initial adjustment are preserved, as sufficient redundancy and geometric strength are important in ensuring correct identification.

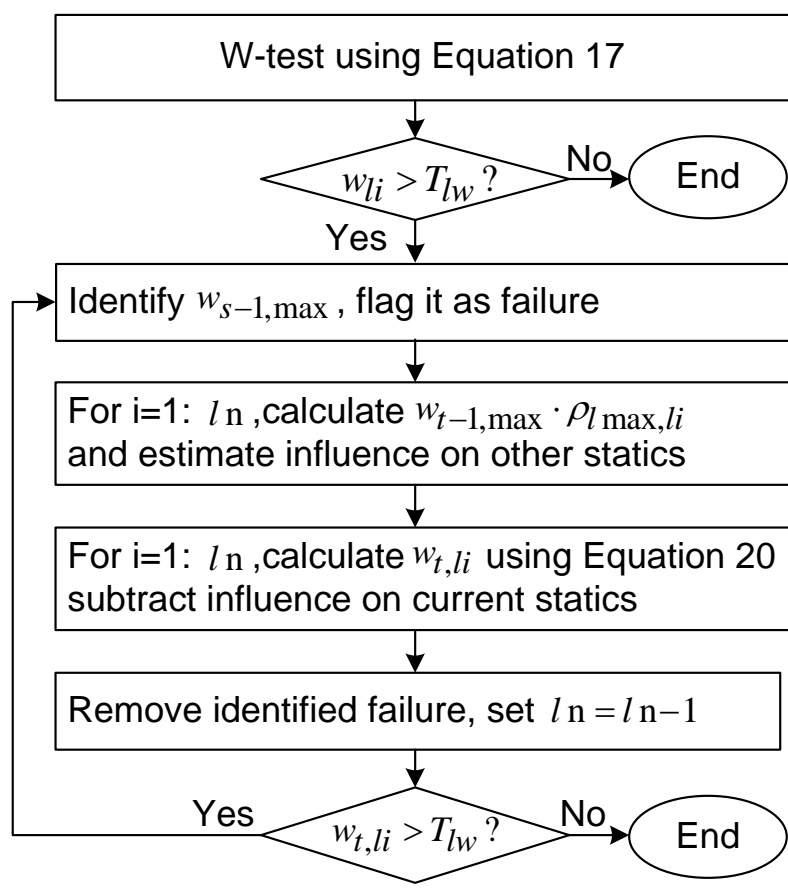

Fig.3 Processing of extended w-test

\section{Demonstration results and analysis}

In order to demonstrate the approach proposed, the cooperative GNSS positioning experiments were carried out at the Nottingham Geospatial Institute (NGI) of the University of Nottingham. Two vehicles and one GNSS reference station are used. Vehicle A is an electric train, whose running track has been scanned with high spec laser scanner to a resolution of $2 \mathrm{~mm}$. Vehicle B is a road vehicle, which moves simultaneously with vehicle A within a relative distance of 100 meters. The GNSS reference station, vehicle A and $\mathrm{B}$ constitute a situation for cooperative positioning, as shown in Figure 4. Both the vehicles A and B are equipped with Leica GS10 GNSS receivers, which can provide dual frequency code and carrier phase GPS and GLONASS data. For vehicle A the previously scanned track is used as the "true" reference, while for vehicle B the high performance INS (Applanix POS/RS with Honeywell C-IMU), wheel odometer and Leica Nova TS50 data are integrated to provide a position solution.

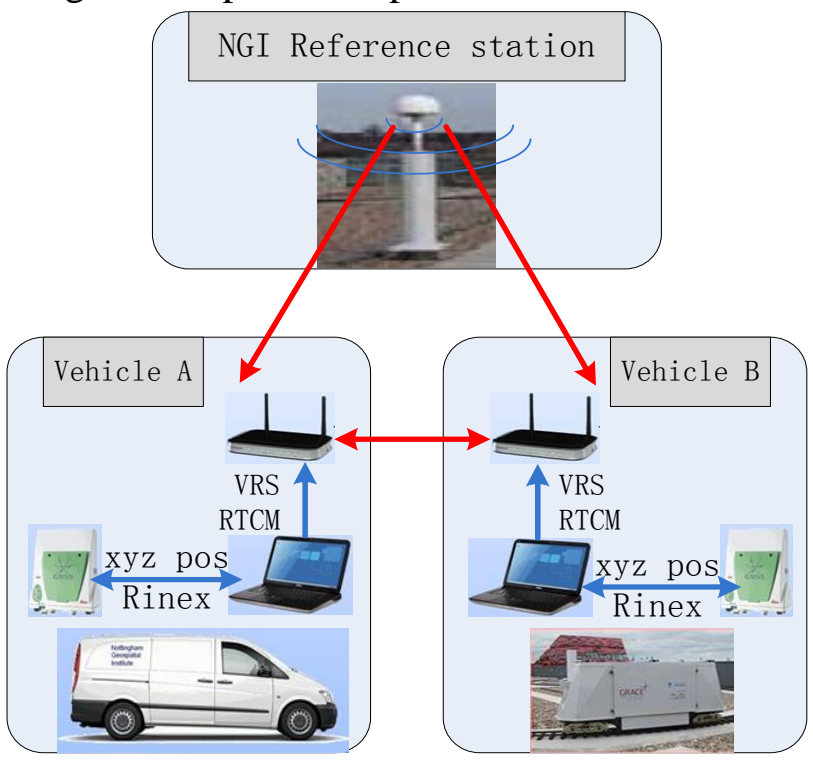

Fig. 4 Cooperative navigation system

In the experiment, vehicle $B$ uses the observations (code and carrier phase) of the reference station and its own measurements to perform the code and carrier phase DD. Once an absolute position of vehicle B can be obtained, it will share its absolute position and some raw RINEX information with vehicle A. Suppose vehicle A cannot form a V2I situation with the reference station due to a blockage or other reasons, it will then use the shared information from vehicle $\mathrm{B}$, together with its own observed RINEX data to carry out cooperative positioning. The absolute position of vehicle $\mathrm{A}$ is calculated based on this V2V system.

The sampling frequency of the raw data for vehicle $\mathrm{A}$ and vehicle $\mathrm{B}$ is $20 \mathrm{~Hz}$ so that different cooperative positioning performance can be analyzed at different sampling rate and delay. The cutoff angle of visible satellites is set to $20^{\circ}$, $P_{F A}$ is chosen as $5 \%$. The series of common visible satellites is shown in Figure 5. As can be seen, satellite 28 appears at $55 \mathrm{~s}$ while satellite 22 becomes unavailable from 69 s. From 69 s to 180 s the set of visible satellite is [ $\begin{array}{lllll}1 & 3 & 11 & 14 & 19\end{array}$ 28 32], and ordered by elevation from high to 
low the sequence is $\left[\begin{array}{lllllll}11 & 1 & 32 & 19 & 14 & 3 & 28\end{array}\right]$, thus satellite 11, whose elevation is the largest, is chosen as the priori satellite.

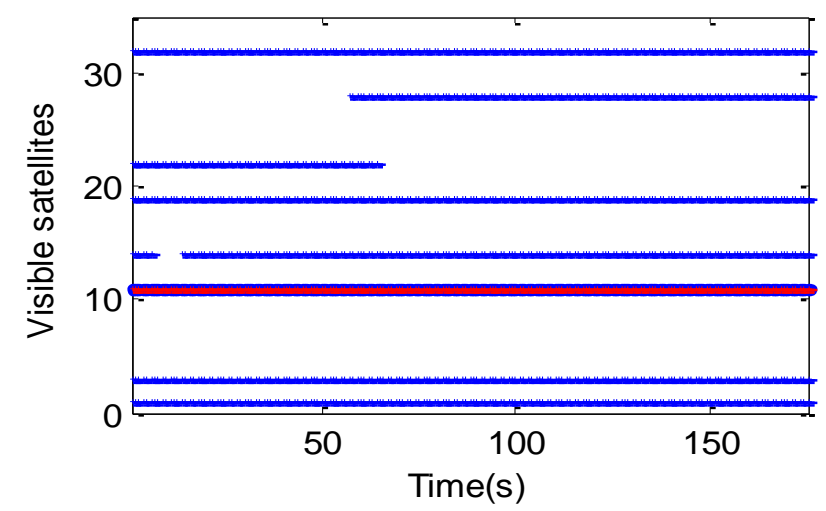

Fig. 5 Common visible satellites

The new closed-loop EKF approach is quantified by comparing three popular methods. The first method is the prominent LS which performs a float estimate in the first step then a LAMBDA to fix ambiguities (Verhagen and $\mathrm{Li}$ 2012), the second method is the normal EKF approach proposed in several relative kinematic positioning applications (Wolfe et al. 2007), and the third method uses differenced code based EKF techniques (Muller et al. 2014). The variance of the code measurements $\sigma_{c}^{2}$ is set to $4 \mathrm{~m}^{2}$ and the variance of the carrier phase measurement is set to $0.0009 \mathrm{~m}^{2}$.

The results of the cooperative positioning are shown in Figure 6. The top panel shows the absolute positions of vehicle A (electric train), road vehicle $B$ and the reference station. The position of the road vehicle is achieved by the new EKF using DD between the reference station and vehicle $\mathrm{B}$. The position of the train is derived from the shared information of the road vehicle position plus the relative position. In the bottom panel, the relative position of vehicle $A$ and $B$ is calculated by the new EKF, LS, normal EKF and code EKF respectively. Their results are compared with the reference (the subtraction between the GNSS/INS tightly coupled results of vehicle B and the previously scanned track of vehicle A). The cooperative positioning results of vehicle A using those methods are shown in the top panel of Figure 7, and their errors are shown in the bottom panel. As can be seen, the new EKF is very consistent with the reference results, which is significantly better than others.

The mean errors (3D) of the new EKF vs. other methods for vehicle $\mathrm{A}$ is shown in Table 1. As can be seen, centimeter-level positioning accuracy can be obtained from cooperative positioning using the new EKF. For the LS method, only decimeter-level accuracy can be obtained. Compared with the normal EKF, the positioning error of new EKF is significantly reduced $(0.023 \mathrm{~m}$ vs. $0.083 \mathrm{~m}$ using dual frequency signal and 0.218 vs. 0.675 using single frequency signal). This is due to the normal EKF requiring that the float ambiguities are sufficiently close to the exact integers, otherwise, the LAMBDA will not be able to fix ambiguities and the positioning accuracy will be degraded. It is also can be seen that the code based EKF is the worst because it sacrifices the higher accuracy available from carrier phase measurements. Meanwhile, the performance of the dual frequency test is significantly higher than the single frequency test for all the methods. This is due to that the success rate for dual frequency carrier phase ambiguity fix is higher than single frequency $(99.48 \%$ vs. 79.67\% using the new EKF).

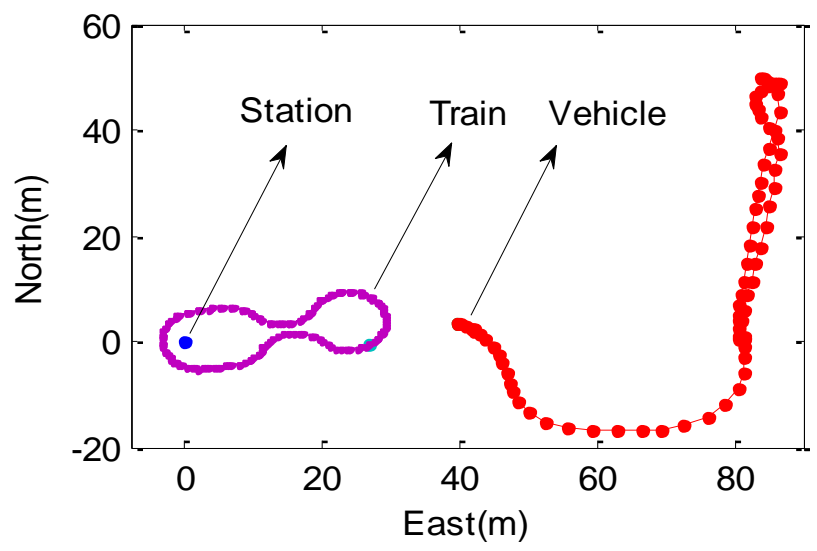




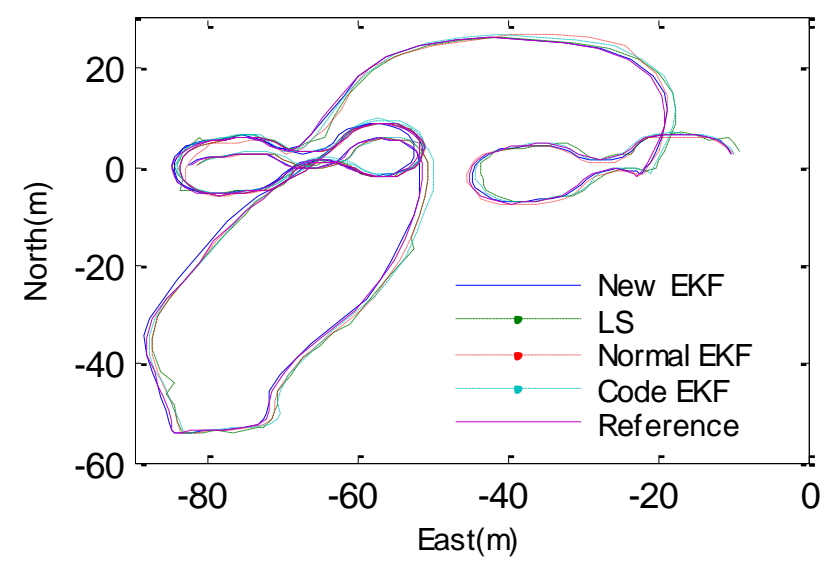

Fig. 6 Results of cooperative positioning. Absolute (top) and relative positions (bottom) for vehicle A and $\mathrm{B}$
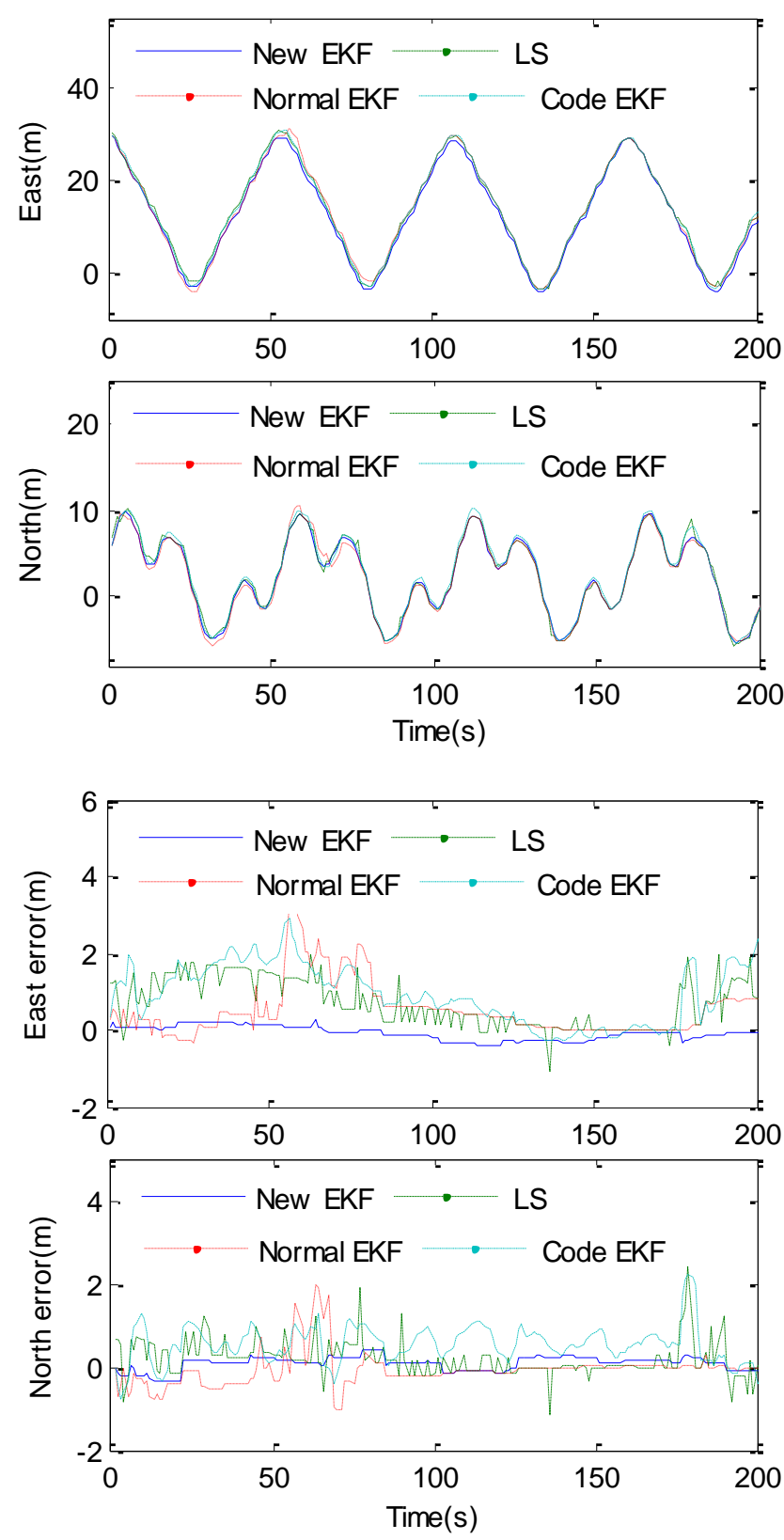

Fig. 7 Cooperative results of vehicle A using different methods. Positioning results (top) and their errors (bottom)

Table 1 Mean errors in meters using different methods

\begin{tabular}{lllll}
\hline Solution & $\begin{array}{l}\text { New } \\
\text { EKF }\end{array}$ & LS & $\begin{array}{l}\text { Normal } \\
\text { EKF }\end{array}$ & $\begin{array}{l}\text { Code } \\
\text { EKF }\end{array}$ \\
\hline L1+L2 & 0.032 & 0.127 & 0.083 & 0.579 \\
L1 & 0.218 & 0.832 & 0.675 & 1.146 \\
\hline
\end{tabular}

The positioning results for using different sampling rates are also compared, which is shown in Table 2. As can be seen, the performance at $10 \mathrm{~Hz}$ and $20 \mathrm{~Hz}$ is similar, where both are slightly better than $1 \mathrm{~Hz}$. This shows that in vehicle applications, high sampling rate usually contributes little in improving the positioning performance, On the contrary it increases the data transfer and recalculation burden.

Table 2 Mean errors in meters using new EKF

\begin{tabular}{llll}
\hline Solution & $1 \mathrm{~Hz}$ & $10 \mathrm{~Hz}$ & $20 \mathrm{hz}$ \\
\hline $\mathrm{L} 1+\mathrm{L} 2$ & 0.032 & 0.028 & 0.027 \\
$\mathrm{~L} 1$ & 0.218 & 0.203 & 0.205 \\
\hline
\end{tabular}

In order to validate the performance of multi-failure diagnosis for new EKF cooperative positioning, two failures are artificially added to the code and carrier phase measurements. Results show that good performance were obtained in both V2I (vehicle $\mathrm{B}$ vs. reference station) and $\mathrm{V} 2 \mathrm{~V}$ (vehicle A vs. vehicle $\mathrm{B}$ ) tests. The $\mathrm{V} 2 \mathrm{~V}$ tests using single frequency data at $1 \mathrm{~Hz}$ is used for demonstration here.

First, two step failures of $25 \mathrm{~m}$ are added to the code measurements of satellites 19 and 32 between 100s and 120s. The failure diagnosis results are shown in Figures 8 and 9, and Table 3. In the top panel of Figure 8, it can be seen that the step failure of code measurements can be detected immediately by the proposed CRAIM method. The $1^{\text {st }}$ iteration of the w-test for code DDs is shown in the middle panel of Figure 8. As can be seen, due to the correlation 
of the statistics, two failures induced the code DDs of satellites 1, 19 and 32 exceed the threshold, where the largest is the $32^{\text {nd }}$ satellite (5.325m as shown in Table 3). The first iteration of the w-test for the carrier phase DDs is shown in the bottom panel of Figure 8, which indicates that the failures of code DDs has no influence on the statistics of the carrier phase DDs. However, it can detect the outliers of satellites 14, 22 and 28, due to the changing of visible satellites as shown in Figure 5. The second iteration of w-test for code DDs and w-test for carrier phase DDs are shown in Figure 9. It can be seen that the code DD of the satellite 19 has the biggest test statistic $(3.526 \mathrm{~m}$ as shown in Table 3), so this satellite is considered as the second failure in this phase. As no more test statistics are seen to exceed the threshold in the third iteration, the iteration is ended and the code DDs of the satellites 32 and 19 are considered as failures from the first two iterations.
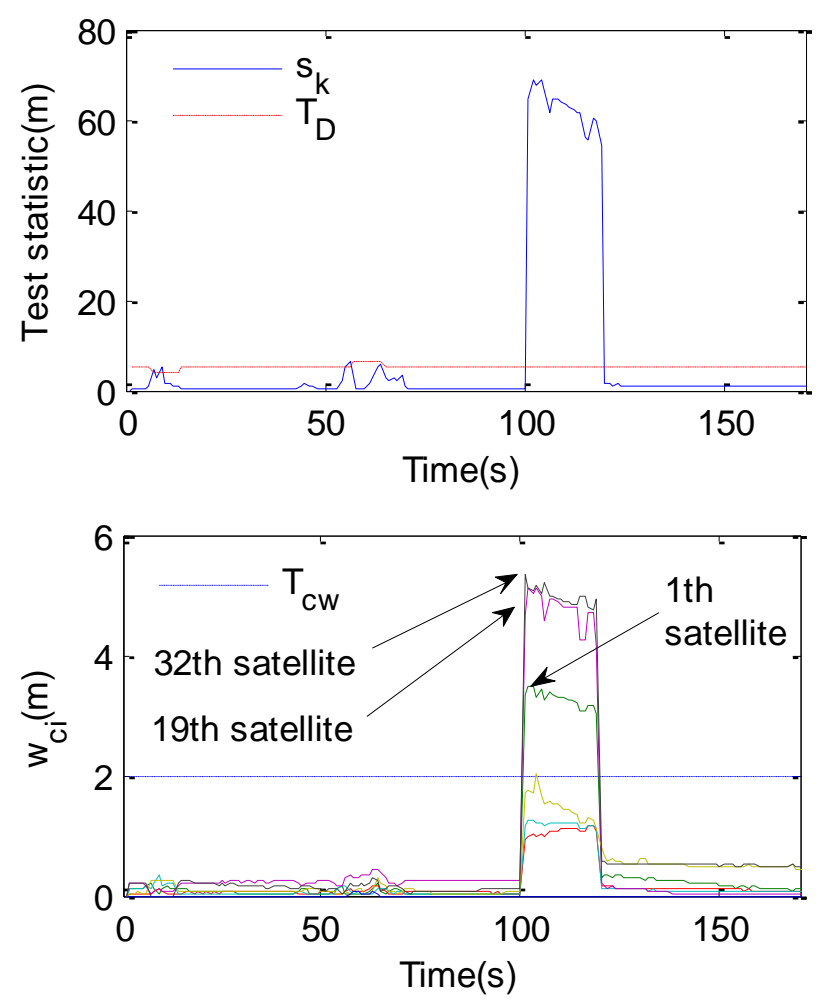

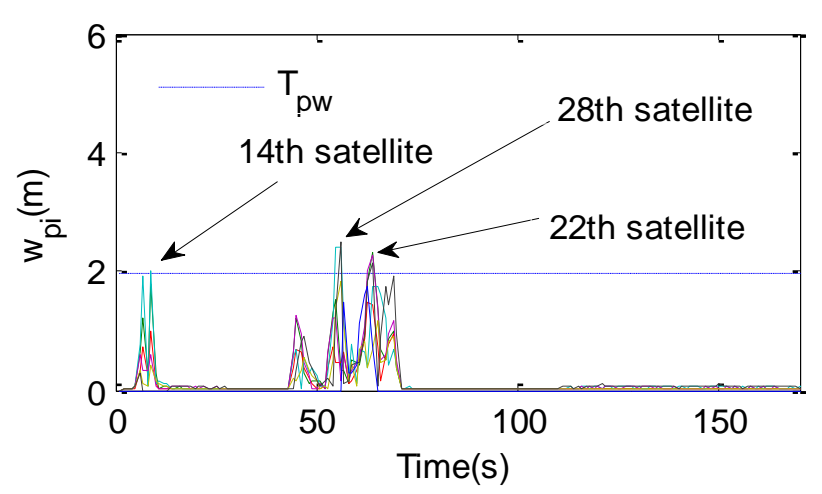

Fig. 8 Step failure diagnosis on code measurements. Detection result (top), the $1^{\text {st }}$ iteration of w-test for code DDs (middle) and carrier phase DDs (bottom)
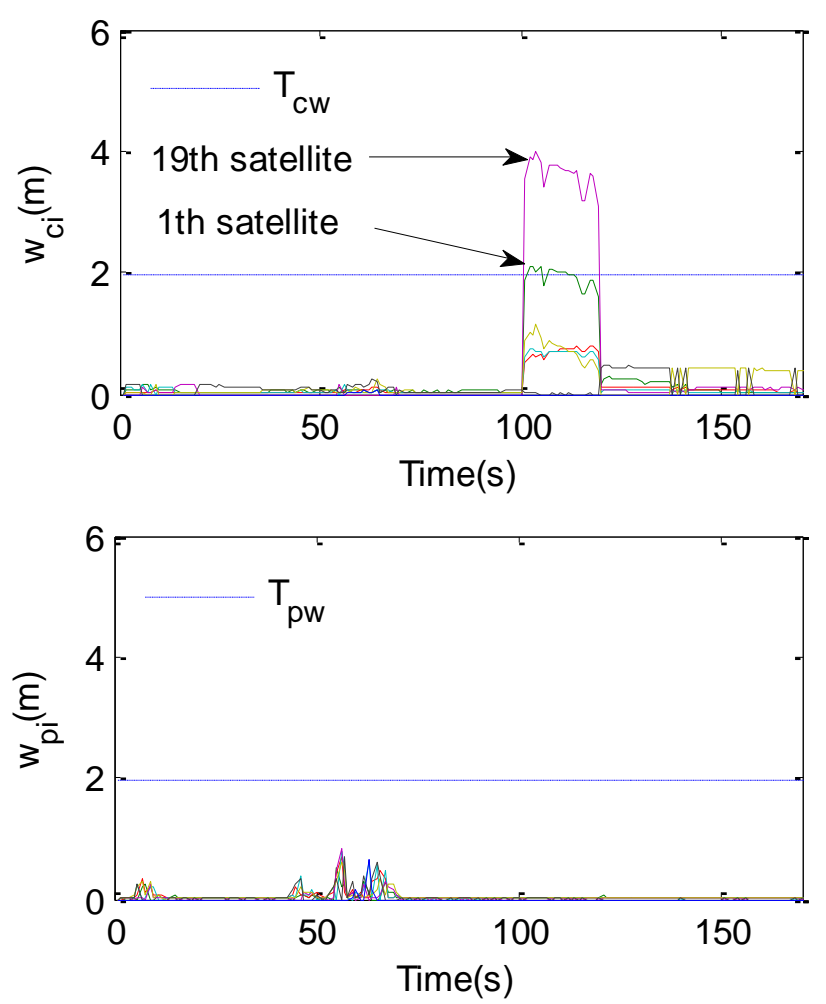

Fig. 9 The second iteration results of step failure diagnosis on code measurements. Code DDs (top) and carrier phase DDs (bottom)

Table 3 Results of code DDs at 120s

\begin{tabular}{llllll}
\hline $\mathrm{i}$ & $\begin{array}{l}\text { DD } \\
\text { pairs }\end{array}$ & $\rho_{i j \max }$ & $w_{1, i}$ & $w_{2, i}$ & $w_{3, i}$ \\
\hline 1 & 11,1 & 0.280 & 3.343 & 1.955 & 0.978 \\
2 & 11,3 & 0.092 & 0.921 & 0.512 & 0.285 \\
3 & 11,14 & 0.114 & 1.166 & 0.613 & 0.320 \\
4 & 11,19 & 0.249 & 4.680 & $\mathbf{3 . 5 2 6}$ & 0.000 \\
5 & 11,28 & 0.149 & 1.683 & 0.889 & 0.411 \\
6 & 11,32 & 0.279 & $\mathbf{5 . 3 2 5}$ & 0.000 & 0.000 \\
\hline
\end{tabular}


For further tests, two slips events of 25 cycles are added to the carrier phase measurements of the satellites 19 and 32 between 100s and 120s. The integrity monitoring results are shown in Figures 10 and 11, and Table 4. Similar to the analysis of code measurement results, the multi-failure of carrier phase measurements can also be detected efficiently with the proposed CRAIM method. Meanwhile, the failures of satellites 19 and 32 can be identified through the iteration of the extended w-test proposed in this study. Furthermore, by comparing Tables 3 and 4 , it can also be seen that the correlation coefficients of carrier phase DD is larger than code DD, which means that the failure identification of carrier phase DD is more difficult when compared with code DD. Nevertheless, the proposed multi-failure diagnosis method is efficient for both code measurements and carrier phase measurements.
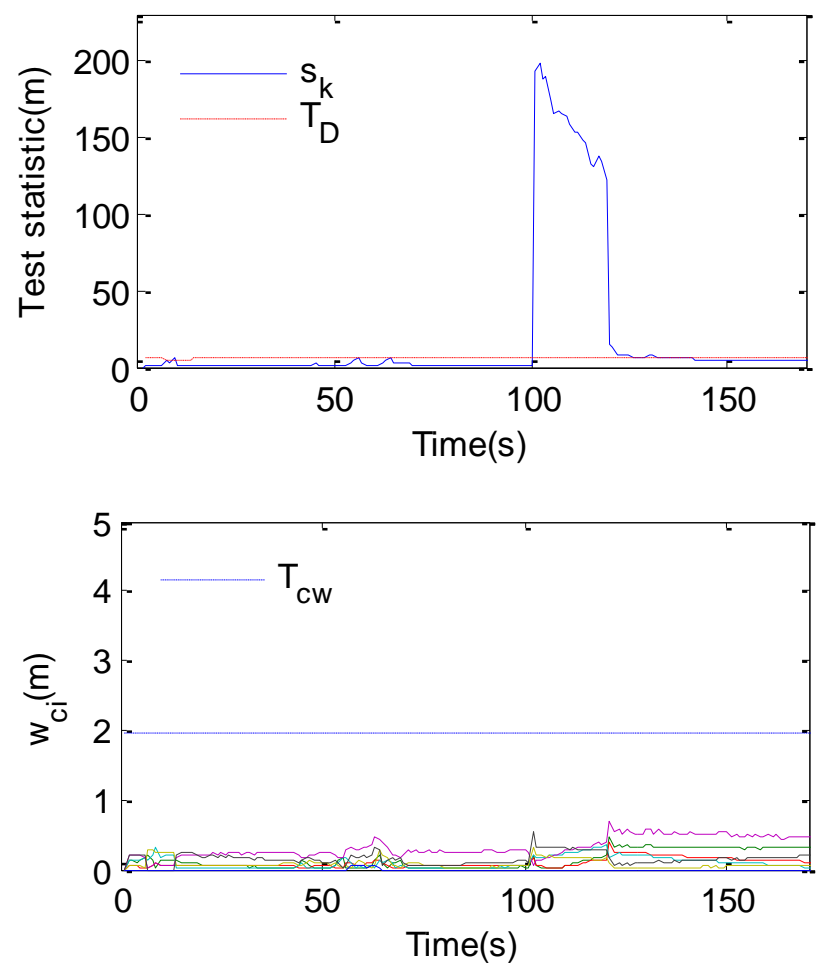

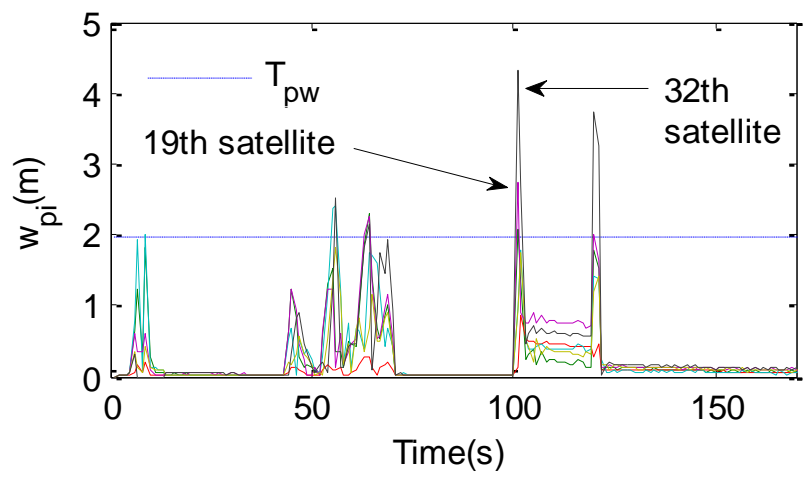

Fig. 10 Step failure diagnosis on carrier phase measurements. Detection result (top), the first iteration of w-test for code DD (middle) and carrier phase DD (bottom)
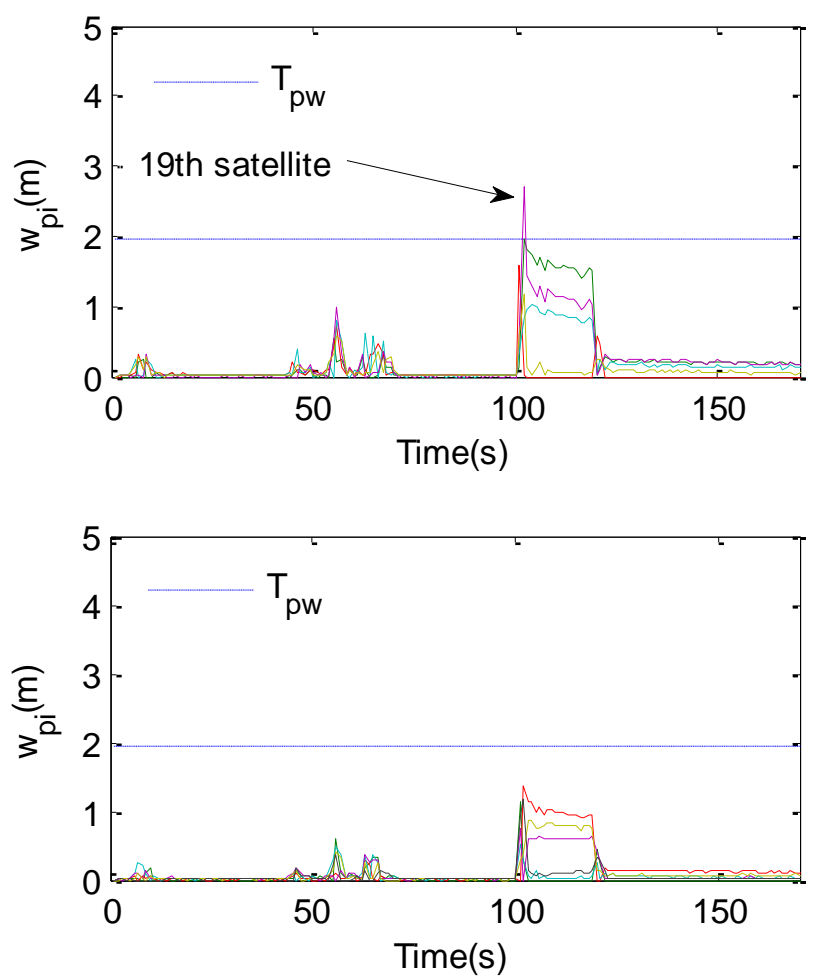

Fig. 11 The second and third iteration results for carrier phase DDs. The second iteration (top) and third iteration (bottom)

Table 4 Results of carrier phase DD at 120s

\begin{tabular}{lccrrr}
\hline No. & $\begin{array}{c}\text { DD } \\
\text { pairs }\end{array}$ & $\rho_{i j \max }$ & $w_{1, i}$ & $w_{2, i}$ & $w_{3, i}$ \\
\hline ph1 & 11,1 & 0.986 & 2.162 & 1.955 & 0.275 \\
ph2 & 11,3 & 0.788 & 0.735 & 0.051 & 1.353 \\
ph3 & 11,14 & 0.566 & 0.825 & 0.747 & 0.304 \\
ph4 & 11,19 & 0.985 & 2.722 & $\mathbf{2 . 2 5 9}$ & 0.000 \\
ph5 & 11,28 & 0.719 & 1.232 & 1.170 & 0.475 \\
ph6 & 11,32 & 0.590 & $\mathbf{3 . 9 3 2}$ & 0.000 & 0.000 \\
\hline
\end{tabular}




\section{Conclusion}

Cooperative GNSS positioning for multi-vehicles has achieved encouraging results in recent years. However, most research is based on sharing code measurements rather than carrier phase measurements. Under these circumstances, the positioning accuracy and integrity cannot meet the high performance requirements of future ITS such as connected vehicles or autonomous driving applications. In order to overcome these problems, a new EKF and a multi-failure diagnosis algorithm are developed to process GNSS pseudorange and carrier phase measurements. First, a new closed-loop EKF with PAR as feedback to address the low accuracy issue is introduced. A multi-failure diagnosis algorithm is then proposed to improve the integrity and reliability. The core of this new algorithm includes using modified CRAIM method for failure detection, and the double extended w-test detectors to identify failures. The effectiveness is demonstrated by a cooperative positioning experiment using a GNSS reference station, an electric train and a road vehicle. High accuracy cooperative positioning results have been achieved, and the multi-failure diagnosis algorithm is validated for both the code and carrier phase measurements.

\section{Acknowledgment}

This research was supported by the Central Universities NUAA Fundamental Research (NS2015087). China Scholarship Council is gratefully acknowledged for supporting the first author's visiting at The University of Nottingham. The authors also gratefully acknowledge the helpful comments and suggestions of the reviewers, which have improved the presentation.

\section{References}

Alam N, Dempster A G (2013) Cooperative positioning for vehicular networks: facts and future. IEEE Transactions on Intelligent Transportation System 14(4):1708-1717

Bauernfeind R, Dötterböck D, Gkougkas E, Eissfeller B (2013) Cooperative GNSS Signal Processing Algorithms for Intelligent Transport Systems. Proc. ION GNSS+ 2013, Institute of Navigation, Nashville, Tennessee, September 16-20, pp.645-655

Basnayake C, Lachapelle G, Bancroft J (2011) Relative positioning for vehicle-to-vehicle communication enabled vehicle safety applications. Proc. of the 18th ITS World Congress, Orlando, Florida, pp.1-16

Eugenio R, Mirko R (2013) GoGPS: open source software for enhancing the accuracy of low-cost receivers by single-frequency relative kinematic positioning. Measurement Science and technology 24(11): 1-12

Feng S J, Ochieng W, Moore T (2009) Carrier phase-based integrity monitoring for high-accuracy positioning. GPS Solutions, 13(1):13-22

Hewitson S, Wang J L (2006) GPS receiver autonomous integrity monitoring (RAIM) for multiple outliers. European Journal of Navigation 4(4): 41-48

Hewitson S, Wang J L (2007) GNSS receiver autonomous integrity monitoring with a dynamic model. The Journal of Navigation 60(2): 247-63

Hewitson S, Wang J L (2010) Extended receiver autonomous integrity monitoring (eRAIM) for GNSS/INS integration. Journal of Surveying Engineering 136(1): 13-22

Hofmann W B, Lichtenegger H, Wasle E (2008) GNSS-Global Navigation Satellite Systems, Springer-Verlag Wien New York

Jokinen A, Feng S J, Ochieng W (2012) Fixed ambiguity Precise Point Positioning (PPP) with FDERAIM. Position Location and Navigation Symposium (PLANS), 2012 IEEE/ION, pp. 643-658

Knight N L, Wang J, Rizos C (2010a) Generalized measures of reliability for multiple outliers. J Geod, Vol.84:625-635

Knight N L, Almagbile A, Wang J, Ding W (2010b) Optimizing fault detection and exclusion in positioning, IEEE Conference of Ubiquitous Positioning Indoor 
Navigation and Location Based Service, Kirkkonummi, Finland, pp.1-10

Lin C W, Jan S S (2014) Algorithm and configuration availability tool for integrity monitoring test bed. Proc. ION GNSS+ 2014, Institute of Navigation, San Diego, California, January 27-29, pp. 223-233

Liu H, Xu L, Ye W (2013) Partial ambiguity resolution with integrity risk constraint for high-performance Global Navigation Satellite System navigation. Proceedings of the Institution of Mechanical Engineers, 2013, DOI: 10.1177/0954410013511427

Liu K, Lim H B, Frazzoli E, Ji H (2014) Improving Positioning Accuracy Using GPS Pseudorange Measurements for Cooperative Vehicular Localization. IEEE transaction on Vehicle technology 63(6):2544-2556

Montenbruck O, Wermuth M, Kahle R (2012) GPS based relative navigation for the TanDEM-X mission - first flight results. Navigation 58(4): 293-304

Muller F P, Diaz E M, Kloiber B, Strang T (2014) Bayesian cooperative relative vehicle positioning using pseudorange differences. IEEE Position, Location and Navigation Symposium, Monterey, CA, pp.434-444

Sabatini R, Moore T, Hill C (2013) A novel GNSS integrity augmentation system for civil and military aircraft. International Journal of Mechanical, Industrial Science and Engineering 7(12): 1486-1452

Schuster W, Bai J, Feng S, Ochieng W (2012) Integrity monitoring algorithms for airport surface movement. GPS Solutions 16(1): 65-75

Stephenson S, Meng X, Moore T (2012) Implementation of V2X with the integration of Network RTK: Challenges and solutions. The 25th International Technical Meeting of the Satellite Division of The ION, Nashville TN, pp.1556-1567

Stephenson S, Meng X, Moore T (2014) A fairy tale approach to cooperative vehicle positioning. Proc. ION GNSS+ 2014, Institute of Navigation, San Diego, California, pp.431-440

Teunissen P J G, Verhagen S (2009) GNSS carrier phase ambiguity resolution: challenges and open problems. International association of geodesy symposia. Observing our Changing Earth, Berlin, pp.785-792
Verhagen S, Li B (2012) LAMBDA-Matlab implementation, version 3.0. Delft University of Technology and Curtin University

Walter T, Blanch J, Enge P (2014) Reduced subset analysis for multi-constellation ARAIM. Proc. ION ITM 2014, Institute of Navigation, San Diego, California, pp. 89-98

Wang H N (2012) GPS navigation principles and applications. Alpha Science International Ltd

Wolfe J D, Speyer J L, Hwang S (2007). Estimation of relative satellite position using transformed differential carrier-phase GPS measurements. Journal of Guidance, Control and Dynamics 30(5):1217-1227

Yun H, Kee C (2014) Multiple-hypothesis RAIM algorithm with an RRAIM concept. Aircraft Engineering and Aerospace Technology 86(1): 26-32

\section{Author Biographies}

Haiying Liu is an Associate Professor of Nanjing University of Aeronautics and Astronautics, Nanjing, China, with MS and Ph.D in Automatic Control. His research interests are Navigation Guidance and control, multisensory integration of GNSS, INS, etc.

Xiaolin Meng is an Associate Professor, Theme Leader for Positioning and Navigation Technologies, and MSc Course Director for GNSST \& PNT at the University of Nottingham. His research interests include ubiquitous positioning, location based services, ITS and services, network real-time kinematic GNSS positioning, etc.

Zhiming Chen is an Assistant Professor of Nanjing University of Aeronautics and Astronautics, Nanjing, China. His research interest focuses on Navigation Guidance and control, satellite navigation, etc.

Scott Stephenson is a doctor student at Nottingham Geospatial Institute of The University of Nottingham. His research interest focuses on cooperative navigation, GNSS technology.

Pekka Peltola is a doctor student at Nottingham Geospatial Institute of The University of Nottingham. His research interest focuses on Navigation and Positioning, multisensory integration. 
